\title{
Vivant Denon's Point de lendemain
}

Henriette JAVOREK

Q

ue peut-on perdre en s'abandonnant à une histoire qui n'a Point de lendemain?' On ne risque qu'à gagner une experience sans durée, mais néanmoins pleine d'avantages. Mme de T..., l'héroïne de Vivant Denon dans la seule nouvelle de cet auteur, entrevoit une telle liaison avec le jeune narrateur, Damon. ${ }^{2} \mathrm{La}$ grande dame trame délicatement une aventure qui la servira à trois reprises. Au lieu d'être obligée de confronter son mari le premier jour de leur réconciliation, elle passe une nuit rêveuse avec un jeune homme qui satisfait ses désirs. En plus, elle dirige la colère et la jalousie de son mari contre Damon, ce qui lui permet de continuer librement ses liens avec le Marquis. Mais endessous de tous ces motifs réside la raison qui pousse le plus Mme de T... vers ses indiscrétions, puisque Mme de T... agit surtout par envie de son amie, la Comtesse. Mme de T..., en essayant d'imiter son amie qu'elle admire et dont elle est jalouse, conçoit un complot d'où elle sort non pas vengée contre tous les joueurs de cette comédie, mais aussi satisfaite et apaisée en elle-même. 
Mme de T... ressent évidemment une grande jalousie envers son amie, la Comtesse. En effet, elle mentionne si souvent la Comtesse que toutes ses actions semblent être dirigées par ce sentiment. La Comtesse figure dans presque toutes les conversations entre Damon et Mme de T... et cette dernière encourage toujours le jeune homme à lui raconter tout ce qu'il sait de la Comtesse. En lui posant des questions comme "où êtes-vous avec mon amie? vous rend-on bien heureux?," Mme de T... manipule toujours le sujet de l'entretient vers la Comtesse (390). Si le jeune homme ne lui répond pas, elle lui reproche, "après avoir risqué de vous priver d'elle, ... Voyez si . . . vous m'avez dit un mot de la Comtesse" (390). Elle lui apprend qu'elle trouve la Comtesse un peu "coquette" et vaine, et demande au jeune homme s'il n'est pas, par hasard, "souvent la victime de cet étrange caractère" (390). ${ }^{3}$ Elle continue "de parler, et toujours de la Comtesse," essayant de renforcer ses propres croyances par le "silence" du jeune homme (391). Même au pavillon, après un moment très tendre, Mme de T... pense toujours à la Comtesse. Elle dit à Damon, "Comme tu sais aimer! Qu'elle est heureuse" (393). Quoique Damon lui demande, "Qui donc? . . . à quel être dans la nature pouvez-vous porter envie," il est évident que Mme de T... envie la Comtesse (393). Un moment plus tard, Mme de T... soulève à nouveau la question de la Comtesse en disant tout simplement, "Mais votre Comtesse" (397). Enfin, après son ultime conquête au château, Mme de T... ose demander ce qu'elle voulait savoir tout au long. Le narrateur décrit la scène: "Elle prit une couronne qu'elle posa sur ma tête, et soulevant à peine ses beaux yeux humides de volupté, elle me dit: 'Eh bien! aimerez-vous jamais la Comtesse autant que moi?'" (398).

$\mathrm{La}$ jalousie que Mme de T... éprouve pour son amie, la Comtesse, se révèle dans toutes les actions et paroles de cette grande dame qui se laisse guider par ses émotions jusqu'à l'achèvement de ses actes prémédités.

Mme de T... suit ses sentiments de jalousie jusqu'au niveau où elle essaie en effet d'imiter la Comtesse, se servant de l'amant 
et de la ruse de cette amie afin de se rendre satisfaite. À la fin de la nouvelle on découvre que Mme de T..., "the woman who, throughout the story ... seems to be following a path she does not know, [is] the instigator of the love scene [and assumes] the position of the secret stage manager" (Reichler 212).

Mais pourquoi Mme de T..., choisit-elle Damon comme sa proie? De quelles secrètes intentions fait-elle preuve afin d'exécuter ses plans? Même si on présume que Mme de T... invente l'intrigue avec l'aide du Marquis, tel un régisseur dans les coulisses, il paraît évident que c'est bien Mme de T... tout au long qui prend les décisions. Quand le Marquis arrive au château le jour après les deux prises d'amour, il explique à Damon qu'on lui "fit dire . . . au moment de [leur] départ" que Mme de T... et le jeune narrateur sont partis (399). Le narrateur se rend compte que le Marquis "venait de débrouiller le mystère de la veille" (399). C'est à dire que le Marquis ne sait pas encore sur qui portera le choix de Mme de T.... Il ne le découvrira qu'après le fait accompli. Mme de T... sélectionne donc Damon toute seule. La jalousie qu'elle ressent envers la Comtesse motive sans doute son choix.

Mme de T..., presque obsédée par la Comtesse, ourdit la tromperie selon le modèle que son amie la Comtesse lui présente à travers ses propres manoeuvres féminines. Mme de T... décrit ainsi les jeux amoureux de son amie: "Combien je lui ai vu jouer de rôles! Entre nous, que de dupes l'environnent! Comme elle s'est moquée du Baron...! Que de tours elle a fait au Marquis" (391).4

Ensuite, elle parle d'un cas en particulier. Elle dit à Damon: Lorsqu'elle vous prit, c'était pour distraire deux rivaux trop prudents et qui étaient sur le point de faire un éclat. Elle les avait trop ménagés, ils avaient eu le temps de l'observer; ils auraient fini par la convaincre. Mais elle vous mit en scène, les occupa de vos soins, les amena à des recherches nouvelles, vous désespéra, vous plaignit, vous consola; et vous fûtes contents tous quatre. (391) 
Cette histoire, ne rappelle-t-elle pas l'intrigue montée par une toute autre héroïne? En effet, Mme de T... donne suite exactement au même complot que son amie. Tout comme chez la Comtesse, quatre personnages figurent dans l'histoire de Mme de T.... Y figurent d'abord le mari et le Marquis, les deux "rivaux" qui chargent Mme de T..., l'un d'une réconciliation et l'autre d'une continuation d'une affaire de coeur illégitime. Les deux autres personnages restent Mme de T... et l'amant de son choix, Damon. Comme la Comtesse, Mme de T... joue "tous, sans rien perdre de la dignité de son caractère" (402). À la fin, ayant accompli ses buts, Mme de T... dit au narrateur:

Adieu, Monsieur; je vous dois bien des plaisirs; mais je vous ai payé d'un beau rêve. Dans ce moment, votre amour vous rappelle; celle qui en est l'objet en est digne. Si je lui ai dérobé quelques transports, je vous rends à elle, plus tendre, plus délicat, et plus sensible. Adieu, encore une fois. Vous êtes charmant... Ne me brouillez pas avec la Comtesse. (402)

Ses projets une fois achevés, Mme de T... peut se permettre toutes les gentillesses au monde. Elle appuie sur sa victoire en rendant Damon à la Comtesse, en le relâchant, usé et point naïf. Elle accueille chaleureusement le Marquis, son amant d'auparavant, sans qu'il se rende compte des infidélités de son amante, ${ }^{5}$ et elle se reconcilie avec son mari, sans contact physique, ayant éliminé les soupçons de ce pauvre homme affaibli. Plus simplement dit, "Tout allait à merveille, et [tous les quatre participants étaient] contents" (401). Tout comme la Comtesse sur qui elle prend exemple, Mme de T... non seulement se fait plaisir mais aussi à tous ceux qu'elle implique dans son jeu.

Une dernière preuve de l'inexprimable désir de Mme de T... de s'élever au niveau de la Comtesse, l'habile et belle manoeuvrière des affaires amoureuses qui garde néanmoins l'estime de tous ses amants, se révèle à travers les deux scènes d'amour, l'une au pavillon et l'autre au château. Au premier instant d'amour, Mme de T... 
se laisse guider par ses émotions, ce qui va tout à fait à l'encontre de ses projets, donc elle retourne à son plan original, l'exécute parfaitement à la bonne fin (comme la Comtesse l'aurait fait), et ainsi, elle accomplit enfin ses buts. Au premier instant d'amour, Mme de T... s'avoue vaincue par sa passion, et ainsi, elle n'avance point ses projets. Même Damon reconnaît que Mme de T... ne veut point nuire à sa réputation en achevant l'aventure qu'elle trame. Le narrateur dit que: "[Mme de T...] semblait avoir quelques projets sur ma personne, mais sans que sa dignité fût compromise. ... Mme de T... avait des principes de décence auxquels elle était scrupuleusement attachée" (385). Mme de T... doit s'attacher à certains "principes de décence," des principes plutôt de vanité pour s'assurer du succès de ses buts. Afin de pouvoir se comparer à la Comtesse, Mme de T... doit non pas séduire le narrateur, mais elle doit aussi rester indifférente au niveau émotionnel. En effet, Mme de T... réussit à n'être point touchée par les hommes jusqu'à ce qu'elle rencontre Damon. Le Marquis, ne dit-il pas qu'elle "fait tout naître, tout sentir, et elle n'éprouve rien: c'est un marbre" (400). Mis à part l'amant de sa rivale, tous les hommes laissent Mme de T... froide. Bien qu'elle charme complètement le jeune homme, le mettant entièrement sous son contrôle, Mme de T... ne supporte pas l'idée qu'elle soit émue par l'amant de la Comtesse. Elle revient alors sur la bonne route, et obtient "une seconde victoire pour s'assurer de sa conquête" (392). Elle entraîne Damon dans les états amoureux encore une fois au château, où, d'après ses projets originaux, elle le séduit sans être le moins du monde touchée elle-même. Évidemment, "ce qui [se passe] entre les deux moments . . . c c'est une augmentation constante de l'artifice," une augmentation du contrôle exercé par Mme de T... (Houppermans $39)$.

Dès le commencement, Mme de T... combat son attirance envers Damon. Le narrateur explique: "Nous nous persuadâmes donc mutuellement qu'il était impossible que nous pussions jamais nous être autre chose que nous nous étions alors" (388). Il est possible que Mme de T... joue ici son rôle parfaitement, convainquant 
le narrateur qu'elle ne pourrait jamais se donner à lui quel que soit la force de son désir, mais il est aussi bien possible qu'elle hésite un peu devant ce jeune homme. Le fait qu'elle éprouve quelques sentiments pour Damon et qu'elle en ait peur devient plus clair dans les quelques scènes suivantes. Mme de T... encourage plusieurs fois le narrateur à rentrer, à suivre le chemin vers le château. Elle dit, "Il faut rentrer, . . . l'air du soir ne nous vaut rien" (389). Elle répète, "rentrons," et encore une fois, "rentrons, je l'exige" (389). Elle veut regagner le chemin qui mène au château afin d'échapper au péril qui la menace dehors où elle ne possède aucune défense. Par conséquent, elle force Damon "de reprendre le chemin du château" (389). Mme de T... essaie de toutes ses forces de rester sur ce chemin, mais elle n'en est pas satisfaite. Le narrateur écrit: "nous cheminions tristement, mécontents l'un de l'autre et de nous-mêmes. . . . nous approchions, occupés en silence de nous soustraire au devoir que nous nous étions imposé si maladroitement" (389-390). C'est à dire que Mme de T... trouve, à ce moment, la réalisation de ses plans peu satisfaisante. En essayant d'accomplir ce devoir "imposé," elle ne ressent que de la tristesse. En effet, en suivant tout de suite le chemin vers le château, Mme de T... manque d'achever un de ses buts principaux; elle manque d'exploiter le sujet de la Comtesse. Comme le narrateur, Mme de T... ne veut point arrêter leur promenade ou leur discussion sur la Comtesse, donc, au dernier moment, presque "à la porte," elle change de direction (390).

Mme de T... et Damon se retrouvent ainsi dans les environs du pavillon où Mme de T... s'égare complètement. Les paroles du narrateur expriment parfaitement les événements qui se passent aussi bien dans le cadre de l'intrigue que dans l'esprit de Mme de T.... Le narrateur dit, "On adore... On ne cédera point... On a cédé" (393). Mme de T... ne prévoit pas l'incident au pavillon. Elle ne projette pas de lui céder au pavillon, mais elle lui cède néanmoins, et elle lui cède complètement. Pour "a woman who is [supposedly] as cold as marble and feels nothing" (Wells 207), Mme de T... manifeste les réactions d'une femme tout à fait muette de saisisse- 
ment. Une fois au pavillon, elle frémit, ses jambes fléchissent, et elle ne peut pas rester debout. Ensuite, elle pose sa main "[1]a main qui voulait [auparavant] repousser [Damon]" (392), sur le coeur du jeune homme. Elle sent battre ce coeur, elle veut le fuir, mais elle finit en retombant, "attendrie" (392). Le narrateur décrit davantage la scène: "Madame de T... se réfugiait dans mes bras, cachait sa tête dans mon sein, soupirait, et se calmait à mes caresses: elle s'affligeait, se consolait, et demandait de l'amour pour tout ce que l'amour venait de lui ravir" (392). Quelle scène! Pourrait-on jamais imaginer la Comtesse dans un tel état de vulnérabilité et de soumission? En se donnant ainsi à Damon, Mme de T... s'éloigne un peu trop de ses projets parce qu'elle risque de paraître faible et de trop se montrer.

Bientôt après la première prise d'amour, Mme de T... se rend compte qu'elle a agit tout à fait au contraire de l'image qu'elle voulait créer, et aussitôt qu'elle se réveille, elle cherche à se sauver. Elle demande, "Sortons de ce dangereux séjour; sans cesse les désirs s'y reproduisent, et l'on est sans force pour leur résister" (393). Si elle espère se comparer à la Comtesse et achever une aventure où elle met en scène quatre personnages dont elle est le maître (elle doit se maîtriser aussi, pour ne pas montrer ses sentiments), elle ne peut point se permettre ce genre d'attendrissement. Elle regrette alors un peu ses faiblesses. Le narrateur remarque: "On parut fâchée de m'avoir affligé, et de s'être laissée emporter trop loin dans une peinture qui pouvait paraître suspecte, étant faite par une femme" (391). Elle se fâche non pas parce qu'elle s'est laissée emporter par ses émotions dans un moment très agréable mais néanmoins nuisible à ses projets, mais parce qu'elle a trop dévoilé sa jalousie de la Comtesse. Elle exprime ses soucis:

Vous m'avez trouvée aimable, et j'aime à croire que vous étiez de bonne foi; mais l'empire de l'habitude est si long à détruire, que je sens moi-même que je n'ai pas ce qu'il faut pour en venir à bout. ... Que pourriez-vous espérer maintenant près de moi? Que pourriez-vous désirer? Et que devient-on avec une 
femme, sans le désir et l'espérance! Je vous ai tout prodigué: à peine peut-être me pardonnerez-vous un jour des plaisirs qui, après le moment de l'ivresse, vous abandonnent à la sévérité des réflexions. (395)

La jalousie que Mme de T... ressent doit rester cachée; Mme de T..., afin de réussir à se mettre au-dessus de tous les personnages de son jeu, doit demeurer le metteur en scène de sa pièce. En plus, pour que son plan marche, Mme de T... doit assurer le bonheur de tous les participants à la fin de l'aventure. Elle doit, pour cette raison, se réjouir d'avoir réalisé ses buts, rejoindre le Marquis, rendre Damon à la Comtesse, et ainsi conserver les sentiments de félicité et de satisfaction pour tous. Donc, après un bref instant de folie, Mme de T... regagne conscience et continue à exécuter son oeuvre de génie.

Mme de T... désire se comporter comme la Comtesse qu'elle décrit ainsi:

Comme elle est fine . . . qu'elle a de grâces! une perfidie dans sa bouche prend l'air d'une saillie; une infidélité paraît un effort de raison, un sacrifice à la décence. Point d'abandon; toujours aimable; rarement tendre, et jamais vraie; galante par caractère, prude par système, vive, prudente, adroite, étourdie, sensible, savante, coquette, et philosophe: c'est un Protée pour les formes, c'est une grâce pour les manières: elle attire, elle échappe. (391)

Par conséquent, Mme de T..., suivant l'exemple de son amie, sort de l'endroit où elle se perd et regagne aussitôt que possible le château où elle peut exercer son contrôle. Elle change sa manière d'agir et de parler, et, tout comme son amie, "toujours aimable," mais "jamais vraie" (391), Mme de T... "devint moins sérieuse" (394). $\mathrm{Au}$ lieu d'éviter le sujet de leur entretien, elle l'explore ouvertement, comme si "une perfidie dans sa bouche [prenait] l'air d'une saillie" (391). Presque étonné, le narrateur déclare qu'elle "osa même plaisanter sur les plaisirs de l'amour, l'analyser, en séparer le 
moral, le réduire au simple, et prouver que les faveurs n'étaient que du plaisir; qu'il n'y avait d'engagement (philosophiquement parlant) que ceux que l'on contractait avec le public, en lui laissant pénétrer [leurs] secrets, et en commettant avec lui quelques indiscrétions" (394). Elle devient donc même "philosophe," exploitant toutes les facettes de leur liaison, mais gardant néanmoins "des grâces" (391). Son langage adopte une finesse subtile quand elle murmure:

Quelle nuit délicieuse ... nous venons de passer par l'attrait seul de ce plaisir, notre guide et notre excuse! Si des raisons, je le suppose, nous forçaient à nous séparer demain, notre bonheur, ignoré de toute la nature, ne nous laisserait, par exemple, aucun lieu à denouer... quelques regrets, dont un souvenir agréable serait le dédommagement... Et puis, au fait, du plaisir, sans toutes les lenteurs, le tracas'et la tyrannie des procédés. (394)

Quoiqu'elle charme encore le narrateur, Mme de T... n'est plus "tendre," mais plutôt "prudente" (391). Elle pense aux conséquences, s'assure du secret de leurs indiscrétions, et prépare le jeune homme au fait qu'il n'y aura point de lendemain. ${ }^{6}$

Mme de T... change complètement de caractère. Elle apprend que "le désir féminin ne connait d'autre loi que celle de l'intempestif dans le devenir imprévisible des aléas du caprice" (Wald-Lasowski 119), donc elle commence à manoeuvrer Damon, à lui murmurer des petites phrases coquettes, à le persuader par la ruse de rentrer au château, et surtout à enfermer Damon à jamais dans sa toile séductrice. Au commencement de cette aventure littéraire, Mme de T... se présente tout à fait naïve; ses efforts pour convaincre Damon à rentrer au château expirent avec quelques soupirs en vain, quelques demandes peu efficaces pour rentrer. Mais après son réveil, Mme de T... ne recourt plus à ces moyens de dilettante; elle ensorcèle le jeune homme et le guide vers le château à son insu. Elle, maintenant "la séductrice[,] guide les pas de son jeune disciple en l'initiant aux secrets d'un univers d'érotisme raf- 
finé" (Herman 239). Elle chuchote tout d'abord:

La belle nuit! . . . les beaux lieux! Il y a huit ans que je les avais quittés; mais ils n'ont rien perdu de leur charme; ils viennent de reprendre pour moi tous ceux de la nouveauté; nous n'oublieront jamais ce cabinet, n'est-il pas vrai? Le château en recèle urı plus charmant encore; mais on ne peut rien vous montrer: vous êtes comme un enfant qui veut touch. er à tout, et qui brise tout ce qu'il touche. (394)

Tout en menant Damon vers le château, Mme de T... s'assure auss. que le jeune homme n'oubliera jamais cette aventure, ce qui fait aussi partie de la ruse de cette dame. Mme de T... dirige donc dès ce moment tous les pas du narrateur. Elle excite "ainsi ... par intervalles [la] curiosité [de Damon] sur [le] cabinet," et elle met le: jeune homme dans l'impossibilité de refuser d'explorer cet endroit mystérieux. En effet, le narrateur reconnaît: "Il faut l'avouer, je ne sentais pas toute la ferveur, toute la dévotion qu'il fallait pour visiter ce nouveau temple; mais j'avais beaucoup de curiosité: ce n'était plus madame de T... que je désirait, c'était le cabinet" (396). Au lieu de chercher à plaire simplement comme une femme, Mme de T... joue un tout autre jeu, plus complexe; 'elle veut que le jeune homme la désire même si elle ne lui plaît plus. C'est à dire qu'elle souhaite se graver dans la mémoire du narrateur comme le symbole d'une femme sollicitée malgré tout, un symbole comme la Comtesse. Ayant déjà "épuisé tout ce que le coeur a de ressources pour enchaîner," Mme de T... adopte les méthodes de son amie et ainsi avance ses plans (395).

Une fois au château, le contraste entre la première scène d'amour et celle au cabinet devient encore plus évident. Tout d'abord, Mme de T... se prépare pour cette reprise. Elle "se débarrassa de tout ornement superflu[,] . . . remplaça tous les . . . ajustements [par] . . . une robe ouverte" pour qu'il n'y ait "pas un noeud à toute [la] parure" (396). On dirait que "les jeux de l'amour laissent à tout moment au hasard," mais Mme de T... ne risque rien; au contraire, elle prévoit même les plus minuscules éléments de la ren- 
contre (Wald-Lasowski 121). Tous ses efforts suscitent du narrateur la réaction suivante: "Enfin elle me parut plus séduisante encore que mon imagination ne se l'était peinte dans nos plus doux moments" (396). Avant de commencer ce qui paraît au narrateur comme "une initiation," Mme de T... s'assure une dernière fois de la sécurité de leurs confidences (396). Elle dit "gravement" au narrateur: "Souvenez-vous, ... que vous serez censé n'avoir jamais vu, ni même soupçonné l'asile où vous allez être introduit. Point d'étourderie; je suis tranquille sur le reste" (396-97). Le calme que Mme de T... manifeste au cabinet ne ressemble point à la façon dont elle tombe en pâmoison au pavillon, saisie de doutes, de tremblements et surtout d'une envie imprévue. Ayant acquis "une autonomie que lui refusaient jusqu'alors les règles rigides et contraignantes du boudoir" (Wald-Lasowski 119), au cabinet, elle, "la reine de ce lieu," conduit Damon dans "une vaste cage" entre des "objets . . . artistement peints," et initie le jeune homme à une allégeance éternelle (397). Une fois complètement préparée, elle se jette "nonchalamment" sur "un dais" où Damon peut tomber "à ses pieds," et elle, en se penchant vers lui et lui tendant les bras, peut consoler ce disciple fraîchement attiré (397). Ce "pacte que les amants ont concerté" est reflété partout dans le cabinet à travers un "miroir [qui, confondant] images et actes vivants, ... . [le] fait refluer de façon idéale à la surface" (Wald-Lasowski 124).

Dans une histoire où, dès le commencement, il n'y existe point de lendemain, "Chacun s'offre volontiers à être la dupe de l'autre et à respecter le prochain, le suivant, afin de ménager sa propre vanité" (Wald-Lasowski 119). Mme de T..., certainement, appartient à ce groupe. Bien qu'elle maîtrise tous les personnages de la nouvelle, elle se montre vulnérable aussi; elle agit par jalousie de la Comtesse. En exécutant ses plans, Mme de T... se dévoile complètement. Elle laisse ressortir sa jalousie envers la Comtesse (elle laisse ressortir même sa colère contre le Marquis puisqu'elle se venge contre cet amant tricheur), et radoucit dans les bras de Damon qui la satisfait là où son mari et le Marquis la déçoivent. Mme de T..., à travers l'aventure qu'elle met en scène, arrive à se débarasser de ses sentiments d'infériorité en face de la ruse et la 
beauté de la Comtesse. En effet, elle devient la reine suprême des affaires amoureuses, digne de l'admiration et du respect de tous. En fin du compte, Mme de T... achève tous ses buts: elle manoeuvre tous les joueurs de sa comédie et les relâche ensuite, satisfaits.

University of Kansas 


\section{Notes}

1 Point de lendemain met un terme à la période d'extase d'une société vouée au plaisir qui, croyant s'être écartée à jamais du Mythe, n'a cessé toutefois de le caresser à travers l'affectation même du dénigrement, perpétuant ainsi, à sa manière, la tradition plusieurs fois séculaire de l'Idéalisme" (Wald-Lasowski 126).

2 "Le nom figure dans le texte de 1777; dans celui de 1812 le protagoniste reste anonyme" (Houppermans 36 ).

3 En effet, Mme de T... arrive, pour un temps, à faire croire au narrateur qu'il éprouve plus d'estime pour elle que pour la Comtesse. Le narrateur dit, "Mon amante [la Comtesse] me parut la plus fausse de toutes les femmes, et je crus tenir l'être sensible [Mme de T...]" (391).

4 Pourquoi Denon, utilise-t-il ce rang social ici quand il a déjà un personnage de ce nom? Il me semble que Denon le fait exprès. À mon avis il veut suggérer une brève liaison entre la Comtesse et le Marquis. Je dirais aussi que ce fait se cache même derrière la jalousie que Mme de T... éprouve pour la Comtesse. Si on accepte ce petit détail, quoique faiblement soutenu, l'intrigue devient tout d'un coup claire. Mme de T..., déjà jalouse de la Comtesse, devient la dupe non pas de son amie mais de son amant, le Marquis aussi. Elle choisit alors de se venger comme la Comtesse le lui avait montré par ses propres actions. Elle prend Damon pour amant, se débarassant ainsi de son mari et de sa fureur contre la Comtesse et le Marquis.

5 Il est évident que le Marquis ignore les actions de Mme de T... puisqu'il dit, "C'est peut-être la femme de Paris sur la fidélité de laquelle il y a le plus à compter," et plus tard, "Tu n'as pas d'idée de son attachement pour moi" (400). Il demande aussi à Damon, "On t'a donc bien pris pour un amant?" (400). Sa question et ses remarques révèlent le fait qu'il s'attendait à une fidélité de Mme de T... et qu'il ne s'apercevait point qu'elle l'avait trompé. Ici, si on accepte la supposition que le Marquis avait, à son tour trompé Mme de T... avec la Comtesse et que Mme de T..., en prenant Damon 
comme son amant, essaie de se venger contre les deux coupables, la victoire de Mme de T... devient encore plus complète.

6 En effet, en ce qui concerne les affaires amoureuses, "nothing lasts until tomorrow" (Diaconoff 262). Un autre critique appuie ce même point, disant que "'Réduits au simple,' les affaires n'ont point de lendemain" (Wald-Lasowski 118), et que "chacun des amants affronte sa propre nuit sans espoir de lendemain" (122). 


\section{Ouvrages Cités}

Denon, Vivant. Point de lendemain. Romanciers du XVIIIe siècle. Vol. 2. Bibliothèque de la Pléiade. Paris: Gallimard, 1965. 379-402.

Diaconoff, Suellen. "The Representation of Desire in Vivant Denon and Watteau." Romance Quarterly 34 (1987): 25973.

Herman, Jan. "Topologie du désir dans Point de lendemain de Vivant Denon." Australian Journal of French Studies 27 (1990): 231-41.

Houppermans, Sjef. "La Description dans Point de lendemain de Vivant Denon." Description-ecriture-peinture. Gronigen: Dept. of French, University of Gronigen, 1987. 36-47. Reichler, Claude. "On the Notion of Intertextuality: The Example of the Libertine Novel," Diogènes Spring-Summer 1981: 205-15.

Wald-Lasowski, Roman. "D'un désir l'autre." Revue des Sciences Humaines. France: (1981): 115-26.

Wells, Byron R. "Objet / volupté: Vivant Denon's Point de lendemain.” Romance Notes 29 (1989): 203-08. 
